\title{
PCS-based structure determination of protein-protein complexes
}

\author{
Tomohide Saio $\cdot$ Masashi Yokochi $\cdot$ \\ Hiroyuki Kumeta $\cdot$ Fuyuhiko Inagaki
}

Received: 31 December 2009/Accepted: 17 February 2010/Published online: 19 March 2010

(C) The Author(s) 2010. This article is published with open access at Springerlink.com

\begin{abstract}
A simple and fast nuclear magnetic resonance method for docking proteins using pseudo-contact shift (PCS) and ${ }^{1} \mathrm{H}^{\mathrm{N}} /{ }^{15} \mathrm{~N}$ chemical shift perturbation is presented. PCS is induced by a paramagnetic lanthanide ion that is attached to a target protein using a lanthanide binding peptide tag anchored at two points. PCS provides long-range $(\sim 40 \AA)$ distance and angular restraints between the lanthanide ion and the observed nuclei, while the ${ }^{1} \mathrm{H}^{\mathrm{N}} /{ }^{15} \mathrm{~N}$ chemical shift perturbation data provide loose contact-surface information. The usefulness of this method was demonstrated through the structure determination of the p62 PB1-PB1 complex, which forms a front-to-back $20 \mathrm{kDa}$ homo-oligomer. As p62 PB1 does not intrinsically bind metal ions, the lanthanide binding peptide tag was attached to one subunit of the dimer at two anchoring points. Each monomer was treated as a rigid body and was docked based on the backbone PCS and backbone chemical shift perturbation data. Unlike NOE-based structural determination, this method only requires resonance assignments of the backbone ${ }^{1} \mathrm{H}^{\mathrm{N}} /{ }^{15} \mathrm{~N}$ signals and the PCS data obtained from several sets of two-dimensional ${ }^{15} \mathrm{~N}$ heteronuclear single quantum coherence spectra, thus facilitating rapid structure determination of the proteinprotein complex.
\end{abstract}

Electronic supplementary material The online version of this article (doi:10.1007/s10858-010-9401-4) contains supplementary material, which is available to authorized users.

\section{T. Saio}

Graduate School of Life Science, Hokkaido University,

Sapporo 001-0021, Japan

T. Saio $\cdot$ M. Yokochi $\cdot$ H. Kumeta $\cdot$ F. Inagaki $(\bowtie)$

Graduate School of Pharmaceutical Sciences,

Hokkaido University, Sapporo 001-0021, Japan

e-mail: finagaki@pharm.hokudai.ac.jp
Keywords Lanthanide-binding peptide tag . Two-point anchoring · Paramagnetic NMR .

Pseudo-contact shift · Rigid-body docking · Autophagy

\section{Introduction}

The structure determination of a protein-protein complex is an important step in revealing the interaction mechanism; however, the application of X-ray crystallography or NMR spectroscopy to this end is not straightforward. In crystallography, complexes are often difficult to crystallize and the possibility of crystal artifacts must always be taken into consideration. In NMR spectroscopy, the protein structure is generally determined on the basis of the short distance restraints derived from nuclear Overhauser effects (NOEs), and it is often difficult to collect a sufficient number of distance restraints for precise structure determination.

Paramagnetic lanthanide ions induce several effects in observed nuclei, such as a pseudo-contact shift (PCS) and residual dipolar coupling (RDC) due to the anisotropy of the magnetic susceptibility tensor ( $\Delta \chi$-tensor). PCS provides long-range distance and angular information between the lanthanide ion and the observed nuclei situated up to $\sim 40 \AA$ apart from the lanthanide ion (Allegrozzi et al. 2000). Accordingly, the paramagnetic lanthanide ion can be used as a powerful probe for solution structure determination, especially for larger molecular weight proteins, multidomain proteins, and protein complexes. For metalloproteins, metal ions such as $\mathrm{Ca}^{2+}$ and $\mathrm{Mg}^{2+}$ can be replaced by the paramagnetic lanthanide ions, and paramagnetic lanthanide probes have been successfully applied to metalloproteins (Bertini et al. 2001, 2004, 2007; Barbieri et al. 2002; Pintacuda et al. 2006, 2007; Allegrozzi et al. 2000). 
However, the application of paramagnetic lanthanide probes to non-metalloproteins requires a general method that attaches the lanthanide ions to the protein at a fixed position. Recently, the lanthanide binding peptide tag (LBT), which can be attached to the target protein by two anchoring points, a disulfide bridge and an N-terminal fusion, has been reported (Saio et al. 2009a). Several other lanthanide-binding tags have been also reported, including lanthanide-chelating reagents attached via disulfide bonds (Dvoretsky et al. 2002; Haberz et al. 2006; Pintacuda et al. 2004; Prudêncio et al. 2004; Ikegami et al. 2004; Leonov et al. 2005; Gaponenko et al. 2002, 2004; Vlasie et al. 2007; Keizers et al. 2007, 2008; Su et al. 2008b), and lanthanidebinding peptides attached through $\mathrm{N}$ - or $\mathrm{C}$-terminal fusion (Gaponenko et al. 2000; Wöhnert et al. 2003; Martin et al. 2007; Ma and Opella 2000; Zhuang et al. 2008) or a disulfide bond (Su et al. 2006, 2008a). However, single-anchored tags tend to be mobile, while symmetrically designed, doubleanchored synthetic chelators often suffer from peak doubling caused by enantiomeric conformers. Furthermore, most of the synthetic tags reported to date are not commercially available. Among these, the lanthanide-attaching method using a two-point anchored peptide tag has a number of advantages in terms of chiral purity, rigidity, and ready availability for protein NMR researchers (Saio et al. 2009a).

We applied this lanthanide tagging method, which introduces the lanthanide ion using two-point anchored peptide tag, to the structure determination of the proteinprotein complex of p62 PB1. P62 is a multi-module adaptor protein that plays an important role in autophagy and the $\mathrm{NF}-\kappa \mathrm{B}$ signaling pathway. In autophagy, p62 interacts with ubiquitinated proteins via its UBA domain, and selfassembles through its PB1 domain to form large protein aggregates (Bjørkøy et al. 2005). The aggregates are then transported to the autophagosome through interaction with LC3 (Noda et al. 2008). The p62 PB1 domain forms a homooligomer in a front-to-back manner using its conserved interaction motifs, the OPCA motif and the conserved Lys motif (Saio et al. 2009b). In order to avoid the homooligomerization of the p62 PB1-PB1 complex, we introduced site-directed mutations into the interaction motifs and prepared two mutants that only limited 1:1 dimer formation. A monomer structure of the p62 PB1 mutant that abrogates homo-oligomerization has been already solved by NMR spectroscopy (Saio et al. 2009b), but the structure of p62 PB1-PB1 complex has not yet been solved. By attaching the lanthanide binding peptide tag to the one subunit of the dimer, we fixed the lanthanide ion on the protein and obtained inter-subunit structural information from the PCS. Here, we demonstrate a simple and fast method for the structure determination of protein-protein complexes in which the monomer structures are docked based on ${ }^{1} \mathrm{H} /{ }^{15} \mathrm{~N}$ PCS and ${ }^{1} \mathrm{H} /{ }^{15} \mathrm{~N}$ chemical shift perturbation data.

\section{Methods}

Plasmid construction

Wild-type p62 PB1 forms a homo-oligomer in front-toback manner, thus making NMR analysis more difficult. On the basis of our previous study (Saio et al. 2009b), we therefore prepared two p62 PB1 mutants, hereafter referred to as $\mathrm{DR}$ and $\mathrm{KE}$, that have mutations in the conserved interaction surfaces, the OPCA motif and the conserved Lys motif, respectively, and thus form a 1:1 dimer. For DR, site-directed mutations were introduced into the conserved acidic residues on the OPCA motif to form a D67A/D69R double mutation. To attach the lanthanide ion to DR, a lanthanide binding sequence comprised of 16 amino acids, CYVDTNNDGAYEGDEL (LBT) (Nitz et al. 2003, 2004; Su et al. 2006, 2008a), was attached to the N-terminus of DR, according to our previous report (Saio et al. 2009a), to which is hereafter referred as LBT-DR. LBT-DR was subcloned, together with a GST tag and a tobacco etch virus (TEV) protease cleavage site, into a pGSTV vector derived from the pET-21 plasmid (Novagen, USA). As a binding partner for LBT-DR, we prepared the KE mutant in which the two basic residues on the conserved basic surface, Lys7 and Arg94, were mutated to Glu and Ala, respectively. The KE mutant was subcloned, with a GST tag and HRV3C protease cleavage site, into a pGSPS vector derived from the pET-21 plasmid (Novagen).

P62 PB1 has two cysteine residues, Cys 26 and Cys42. We changed Cys42 on LBT-DR, and Cys26 and Cys42 on $\mathrm{KE}$ to serine in order to guarantee proper $\mathrm{S}-\mathrm{S}$ formation between LBT and the Cys26 on DR.

\section{Sample preparation}

Proteins were expressed in E. coli strain BL21 (DE3) cells. For the unlabeled samples, cells were grown in Luria-Bertani media. For the uniformly ${ }^{15} \mathrm{~N}$ - or ${ }^{13} \mathrm{C} /{ }^{15} \mathrm{~N}$-labeled samples, cells were grown in M9 media containing ${ }^{15} \mathrm{NH}_{4} \mathrm{Cl}$ $(1 \mathrm{~g} / \mathrm{l})$, Celtone-N powder $(0.2 \mathrm{~g} / \mathrm{l})$ (Cambridge Isotope Laboratories, USA) and unlabeled glucose $(10 \mathrm{~g} / \mathrm{l})$, or ${ }^{15} \mathrm{NH}_{4} \mathrm{Cl}(1 \mathrm{~g} / \mathrm{l})$, Celtone-CN powder $(0.2 \mathrm{~g} / \mathrm{l})($ Cambridge Isotope Laboratories, USA) and $\left[\mathrm{U}_{-}{ }^{13} \mathrm{C}\right]$ glucose $(2 \mathrm{~g} / \mathrm{l})$, respectively. The uniformly ${ }^{15} \mathrm{~N} /{ }^{2} \mathrm{H}$-labeled sample was prepared by culturing cells in $100 \%{ }^{2} \mathrm{H}_{2} \mathrm{O}$ M9 medium using ${ }^{15} \mathrm{NH}_{4} \mathrm{Cl}$ and $\left[\mathrm{U}_{-}^{2} \mathrm{H}\right]$ glucose as the sole nitrogen and carbon sources. Cells were grown at $37^{\circ} \mathrm{C}$ to $\mathrm{A}_{600}$ of 0.8 , and protein expression was induced by the application of Isopropyl $\beta$-D1-thiogalactopyranoside to a final concentration of $0.5 \mathrm{mM}$ for $16 \mathrm{~h}$ at $25^{\circ} \mathrm{C}$. For the preparation of amino acid selectively ${ }^{15} \mathrm{~N}$-labeled samples, the cells were grown at $37^{\circ} \mathrm{C}$ in 11 of minimal media supplemented with $1 \mathrm{~g}^{14} \mathrm{NH}_{4} \mathrm{Cl}$ and $200 \mathrm{mg}$ of 19 unlabeled amino acids, respectively. Protein 
expression was induced at $\mathrm{A}_{600}$ of 0.8 by the addition of isopropyl $\beta$-D-1-thiogalactopyranoside to a final concentration of $0.5 \mathrm{mM}$ and was cultured for $8 \mathrm{~h}$ at $25^{\circ} \mathrm{C}$. Fifty $\mathrm{mg}$ of specific ${ }^{15} \mathrm{~N}$-labeled amino acid was added to the medium 15 min before induction.

For the preparation of LBT-DR, the disrupted cells were centrifuged and the supernatant was applied to glutathioneSepharose 4B resin (GE Healthcare, UK) for affinity purification. The GST tag was removed by incubation for $4 \mathrm{~h}$ at room temperature with TEV protease. The isolated protein was further purified by gel filtration chromatography on a Superdex 75 column (GE Healthcare). LBT-DR was also expressed in the inclusion body, and retrieved by high-pressure refolding (Schoner et al. 2005; Qoronfleh et al. 2007). Details of the refolding process will be published elsewhere. KE was prepared from the soluble fraction, according to the procedure described previously (Saio et al. 2009b).

After the gel filtration, LBT-DR was incubated with $1 \mathrm{mM} \mathrm{5,5'-ditiobis(2-nitrobenzoic} \mathrm{acid)} \mathrm{(DTNB)} \mathrm{for} 2 \mathrm{~h}$ at room temperature, which linked the N-terminal Cys of LBT and the C26 on DR via an intramolecular disulfide bond (Saio et al. 2009a). The oxidized LBT-DR was then mixed with KE, followed by gel filtration chromatography on a Superdex 75 column.

\section{NMR spectroscopy}

For NMR measurements, the samples were prepared in $20 \mathrm{mM}$ MES buffer (pH 6.5) with $50 \mathrm{mM} \mathrm{NaCl}$. All NMR experiments were run on Inova 800, 600 or $500 \mathrm{MHz}$ NMR spectrometers (Varian, USA) at $25^{\circ} \mathrm{C}$. Spectra were processed using the NMRPipe program (Delaglio et al. 1995) and data analysis was performed with the help of the Olivia program developed in our laboratory (Yokochi et al. http://fermi.pharm.hokudai.ac.jp/olivia/). Intermolecular NOEs were obtained from a 3D ${ }^{15} \mathrm{~N}$-edited NOESY experiment with a mixing time of $200 \mathrm{~ms}$ on ${ }^{15} \mathrm{~N} /{ }^{2} \mathrm{H}-$ labeled LBT-DR complexed with unlabeled KE.

Tensor calculation

$\Delta \chi$-tensors for LBT-DR were calculated from the PCS values, and the structure of DR was calculated using the Numbat program (Schmitz et al. 2008) by the equation

$\Delta \delta^{\mathrm{PCS}}=\frac{1}{12 \pi r^{3}}\left[\Delta \chi_{\mathrm{ax}}\left(3 \cos ^{2} \partial-1\right)+\frac{3}{2} \Delta \chi_{\mathrm{rh}} \sin ^{2} \partial \cos 2 \phi\right]$,

where $\Delta \delta^{\mathrm{PCS}}$ is the pseudo contact shift, $r, \partial$ and $\varphi$ are polar coordinates of the nucleus with respect to the principal axes of the magnetic susceptibility tensor, and $\Delta \chi_{\mathrm{ax}}$ and $\Delta \chi_{\mathrm{rh}}$ are the axial and rhombic components, respectively, of the anisotropic magnetic susceptibility tensor. Conformer 1 of the family of NMR structures of DR (PDB code: $2 \mathrm{KKC}$ ) was used for the tensor fit.

\section{Docking}

PCS-based rigid body docking was carried out using the Xplor-NIH program (Schwieters et al. 2003, 2006), equipped with PARA restraints for Xplor-NIH (Banci et al. 2004). The coordinates of LBT-DR (including the metal) were held fixed, whereas KE was treated as a rigid body. As the starting structure of DR, conformer 1 of the family of NMR structures of DR (PDB code: $2 \mathrm{KKC}$ ) was used, with the exception that Cys42 was replaced with Ser using the PyMOL program (http://www.pymol.org/). The structure of the KE mutant was built based on the structure of DR, with the six surface residues of DR (Lys7, Cys 26, Cys 42, Ala67, Arg69, and Arg94) changed to Glu, Ser, Ser, Asp, Asp, and Ala, respectively, in accordance with the amino acid sequence of KE.

At the start of the docking calculation, the relative orientation and position of $\mathrm{KE}$ were randomized to generate 100 starting structures that were located within $100 \AA$ from the DR mutant. The coordinates of DR and the metal, on the other hand, were fixed, with the position of the metal determined by tensor-fits from PCSs observed for LBT-DR. Next, the rigid body docking calculation was performed based on the PCS and contactsurface restraints. During the calculation, the coordinates of DR and the metal were fixed, whereas those of $\mathrm{KE}$ were freely rotated and translated. For the PCS restraints, a pseudo atom representing the tensor axis was introduced. The atom representing the origin of the axis was restrained within $0.3 \AA$ of the metal, while the coordinates of the tensor were freely rotated around the origin. The target function was calculated based on three terms: a square-well quadratic term for ambiguous distance restraints $\left(E_{N O E}\right.$; Clore and Schwieters 2003), the least square energy penalty for PCS restrains $\left(E_{P C S}\right.$; Banci et al. 2004), and a quartic van der Waals repulsion term $\left(E_{\text {repel }}\right)$. Ambiguous distance restraints were set with upper-limit of $5 \AA$. During the minimization process, the force constant for $E_{N O E}$ and $E_{P C S}$ were held constant at $0.01 \mathrm{kcal} \mathrm{mol}^{-1} \AA^{-2}$ and $0.8 \mathrm{kcal} \mathrm{mol}^{-1} \mathrm{ppm}^{-2}$, respectively. The force constraint for $E_{\text {repel }}$ was geometrically increased over 14 cycles from 0.004 to $1 \mathrm{kcal}$ $\mathrm{mol}^{-1} \AA^{-4}$. The van der Waals radius scale factor was decreased from 1.0 to 0.78 . The Xplor-NIH script for the docking calculation is provided as Supporting information. 


\section{Results and discussion}

Construct design for LBT attachment

A lanthanide binding sequence, CYVDTNNDGAYEGDEL (LBT) (Nitz et al. 2003, 2004; Su et al. 2006, 2008a), was introduced to DR according to the procedure reported previously (Saio et al. 2009a). In the DR structure (PDB code: $2 \mathrm{KKC}$ ), the distance between the $\mathrm{C} \alpha$ atoms of Ser3 and Cys26 is around $5 \AA$, which is roughly consistent with the $\mathrm{C} \alpha$ distance between the $\mathrm{N}$ - and $\mathrm{C}$-terminal residues of LBT (PDB code: 1TJB, Nitz et al. 2004), thus we fused the LBT sequence to the N-terminus of Ser3 with a threeresidue linker sequence (His-Met-Gly). To assess the effects of the LBT attachment, we compared the backbone chemical shifts of the ${ }^{15} \mathrm{~N}$-labeled LBT-DR/unlabelled KE containing $1 \mathrm{eq} \mathrm{Lu}^{3+}$ and ${ }^{15} \mathrm{~N}$-labeled DR/unlabelled $\mathrm{KE}$ complexes (Fig. 1). Those residues with chemical shifts significantly affected by the introduction of LBT were found to be located around the anchoring points, while other residues showed only negligible chemical shift changes, indicating that the DR structure was maintained in LBT-DR.

\section{Resonance assignment and PCS measurement}

For the backbone amide resonance assignment of the LBTDR/KE complex, a standard set of triple resonance NMR spectra was measured using the ${ }^{13} \mathrm{C}^{15} \mathrm{~N}$-labeled LBT-DR/ unlabelled $\mathrm{KE}$ and unlabelled LBT-DR $/{ }^{13} \mathrm{C}^{15} \mathrm{~N}$-labeled $\mathrm{KE}$ complexes, both of which contain 1 eq diamagnetic

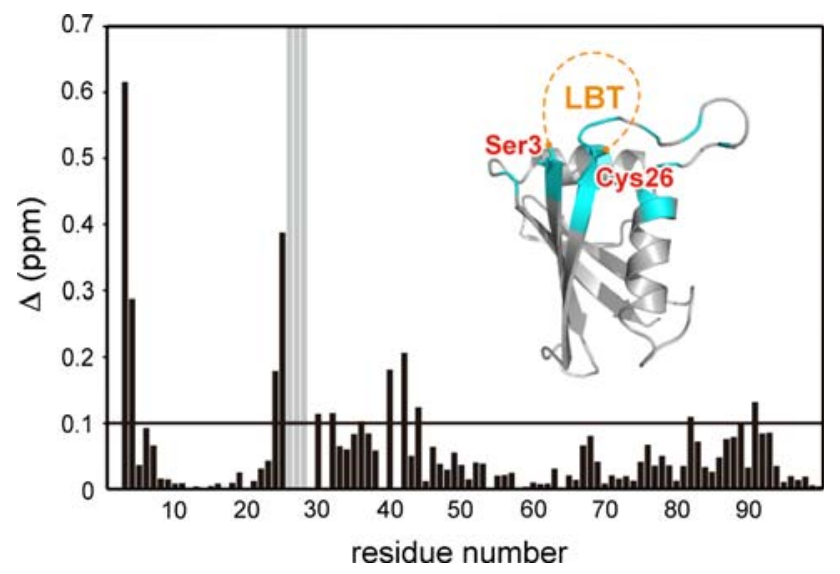

Fig. 1 Chemical shift differences in the backbone amide groups of DR between ${ }^{15} \mathrm{~N}$-labeled LBT-DR/unlabeled KE containing 1 eq $\mathrm{Lu}^{3+}$ and ${ }^{15} \mathrm{~N}$-labeled DR/unlabeled KE. $\Delta(\mathrm{ppm})$ was defined as $\left(\left(\Delta^{1} \mathrm{HN}\right)^{2}+\left(\Delta^{15} \mathrm{~N} / 5\right)^{2}\right)^{1 / 2}$ and plotted against the residue number of DR. The residues for which the signals disappeared upon the introduction of LBT are represented as gray bars. The residues for which the signals shifted $(\Delta \mathrm{ppm}>0.1)$ or disappeared are mapped onto the structure of p62 PB1 lanthanide $\mathrm{Lu}^{3+}$. Resonance assignment was accomplished with reference to those of free-state DR (Saio et al. 2009b). The assignment rate of the backbone amide signals of LBT-DR and KE was 91 and 98\%, respectively.

${ }^{1} \mathrm{H}_{-}{ }^{15} \mathrm{~N}$ HSQC spectra of the ${ }^{15} \mathrm{~N}$-labeled LBT-DR/ unlabelled $\mathrm{KE}$ and unlabelled LBT-DR $/{ }^{15} \mathrm{~N}$-labeled $\mathrm{KE}$ complexes were recorded in the presence of 1 equivalent of lanthanide ions $\left(\mathrm{Lu}^{3+}, \mathrm{Tb}^{3+}, \mathrm{Dy}^{3+}, \mathrm{Er}^{3+}\right.$ and $\left.\mathrm{Tm}^{3+}\right)$, where $\mathrm{Lu}^{3+}$ was used as a diamagnetic reference (Fig. 2a, b). Since the ${ }^{1} \mathrm{H}$ and ${ }^{15} \mathrm{~N}$ of each amide group are spatially close, the PCS has similar ppm values in both ${ }^{1} \mathrm{H}$ and ${ }^{15} \mathrm{~N}$ dimensions (Saio et al. 2009a). Thus, by overlaying the spectra recorded with different lanthanide ions, the signals were found to be located in a straight line. Based on this, the ${ }^{1} \mathrm{H}_{-}{ }^{15} \mathrm{~N}$ HSQC cross-peaks of the paramagnetic samples could be readily assigned using the assignment of the diamagnetic samples. Most PCS-induced resonances for $\mathrm{KE}$ were assigned based on the above procedure. On the other hand, the PCS-based assignment of LBT-DR was not so straightforward due to the complexity of the PCSinduced spectra resulting from the spatial proximity of LBT-DR to the lanthanide ion (Fig. 2a). For reliable PCS assignments for the LBT-DR mutant, amino acid selective ${ }^{15} \mathrm{~N}$-labeled LBT-DR samples were prepared by the selective ${ }^{15} \mathrm{~N}$ labeling of $\mathrm{Arg}$, Phe, or Leu. The ${ }^{1} \mathrm{H}-{ }^{15} \mathrm{~N}$ HSQC spectra of these three samples were recorded in the presence of 1 equivalent of lanthanide ions $\left(\mathrm{Lu}^{3+}, \mathrm{Tb}^{3+}\right.$, $\mathrm{Dy}^{3+}, \mathrm{Er}^{3+}$ or $\mathrm{Tm}^{3+}$ : Fig. 2c and supporting information Figure S1). In case of the ${ }^{15} \mathrm{~N}$-Arg-labeled LBT-DR/unlabeled KE complex, all resonances of the arginine residues in the LBT-DR mutant were assigned (Fig. 2c). The reliable assignments obtained using the amino acid selective labeling were used for further assignments of the uniformly ${ }^{15} \mathrm{~N}$-labeled LBT-DR mutant samples. Finally, a total of 205 PCS-shifted signals for LBT-DR were assigned (38, 49, 58, and 60 signals from $\mathrm{Dy}^{3+}-, \mathrm{Tb}^{3+}-, \mathrm{Er}^{3+}$, and $\mathrm{Tm}^{3+}$-containing samples, respectively), whereas a total of 316 PCS-shifted signals were assigned for $\operatorname{KE}(75,79,82$, and 80 signals from $\mathrm{Dy}^{3+}{ }_{-}, \mathrm{Tb}^{3+}{ }_{-}, \mathrm{Er}^{3+}{ }_{-}$, and $\mathrm{Tm}^{3+}{ }_{- \text {con- }}$ taining sample, respectively: supporting information Table S1).

Determination of the metal position

For the PCS-based docking calculation, the position of the lanthanide ion relative to the coordinates of $\mathrm{DR}$ is required. As the structure of p62 PB1 containing LBT and the metal has not been determined, the metal position was determined based on the backbone amide proton PCS of ${ }^{15} \mathrm{~N}$ labeled LBT-DR/unlabeled KE in complex with $\mathrm{Dy}^{3+}$, $\mathrm{Tb}^{3+}, \mathrm{Er}^{3+}$ and $\mathrm{Tm}^{3+}$, using the numbat program (Schmitz et al. 2008); Based on the PCS values from the four lanthanide ions, $\Delta \chi$-tensors for each lanthanide were 

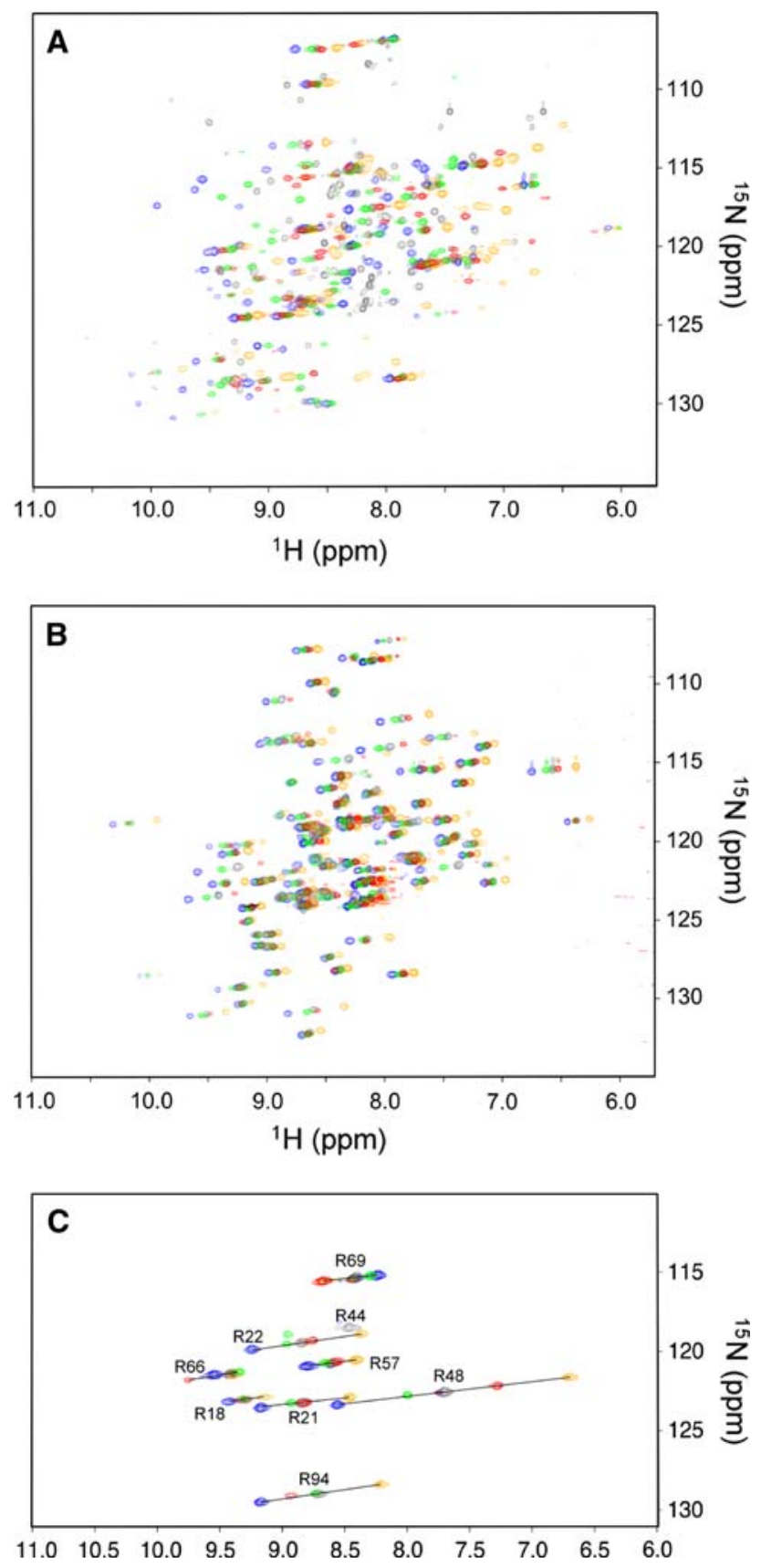

Fig. $2{ }^{1} \mathrm{H}_{-}{ }^{15} \mathrm{~N}$ HSQC spectra of ${ }^{15} \mathrm{~N}$-labeled LBT-DR/unlabeled KE (a), ${ }^{15} \mathrm{~N}$-labeled KE/unlabeled LBT-DR (b), and ${ }^{15} \mathrm{~N}$-Arg-labeled LBT-DR/unlabeled KE (c) in complex with $\mathrm{Lu}^{3+}$ (gray), $\mathrm{Tb}^{3+}$ (orange), $\mathrm{Dy}^{3+}$ (red), $\mathrm{Er}^{3+}$ (green), and $\mathrm{Tm}^{3+}$ (blue). Spectra were obtained using 800 (a and b) and $600 \mathrm{MHz}$ (c) NMR spectrometers at $25^{\circ} \mathrm{C}$

simultaneously fitted as well as a conserved metal position due to their isomorphous nature. Subsequently, the $\Delta \chi$-tensor parameters were further refined for each lanthanide ion (Fig. 3a-c; Table 1), where the position of the metal was allowed to vary within a range of $\pm 0.3 \AA$. The magnitude of the refined tensor was almost the same as that before refinement: within $10 \%$ difference (data not shown).
The $\Delta \chi$-tensor parameters were well defined with the principal axes for the four lanthanides oriented in similar directions and the correlations between the experimental and back-calculated PCS values were good (Fig. 3a, b, and supporting information Figure S2 and S3). It should also be noted that the magnitude of the tensor was comparable to that reported previously (for LBT attached to GB1; Saio et al. 2009a). Thus, we concluded that the position of the lanthanide ion as well as the $\Delta \chi$-tensor parameters were accurately determined. The $\Delta \chi$-tensor parameters were used as an input parameter for docking calculations.

The contact-surface restraints

The DR and KE mutants were docked based on the PCS and contact-surface restraints. The contact surface restraints were generated from the ${ }^{1} \mathrm{H}^{\mathrm{N}} /{ }^{15} \mathrm{~N}$ backbone chemical shift differences between the free and bound states of KE (Fig. 3d). The chemical shift of backbone ${ }^{1} \mathrm{H}^{\mathrm{N}} /{ }^{15} \mathrm{~N}$ is sensitive to the chemical environment of the two nuclei, which is very useful for the identification of the interaction surface on proteins. Unlike NOE-based analysis, the chemical shift perturbation of the backbone signals can easily be obtained without time-consuming side-chain assignment. Combined with the backbone PCS restraints, backbone chemical shift perturbation mapping ensures fast and reliable structure determination of protein-protein complexes. However, chemical shift perturbations can result either from a direct ligand interaction or from a conformational rearrangement around the observed nuclei, and it is possible that the signals of the residue on the opposite side of the interaction surface induce sizable perturbations, as a consequence of a change in the local structure. Thus, we selected interfacial residues according to the three criteria proposed by Clore and Schwieters (2003): (A) significant chemical shift perturbation is observed upon complex formation, (B) at least one or two atoms of the residue are exposed on the surface of the protein, and (C) the selected residue is involved in a cluster of residues on a contiguous, single binding surface. On binding with LBT-DR, several ${ }^{1} \mathrm{H}_{-}{ }^{15} \mathrm{~N}$ HSQC signals for $\mathrm{KE}$ indicated significant chemical shift perturbations (Fig. 3d, e). Asp67, Glu68, Asp69, Asp71, Val73, Phe75, Ser76, Ser77, and Asp90 all indicated large chemical shift perturbations. The eight residues other than Asp90 fulfilled the above-mentioned criteria, whereas Asp90, which indicated a sizable chemical shift difference and whose atoms are exposed on the surface of the protein, failed to comply with criterion C in that Asp90 is located on the opposite side of the continuous cluster comprised of the other eight residues. Thus, we concluded that Asp67, Glu68, Asp69, Asp71, Val73, Phe75, Ser76, and Ser77 are involved in the binding surface, and we converted the chemical shift 

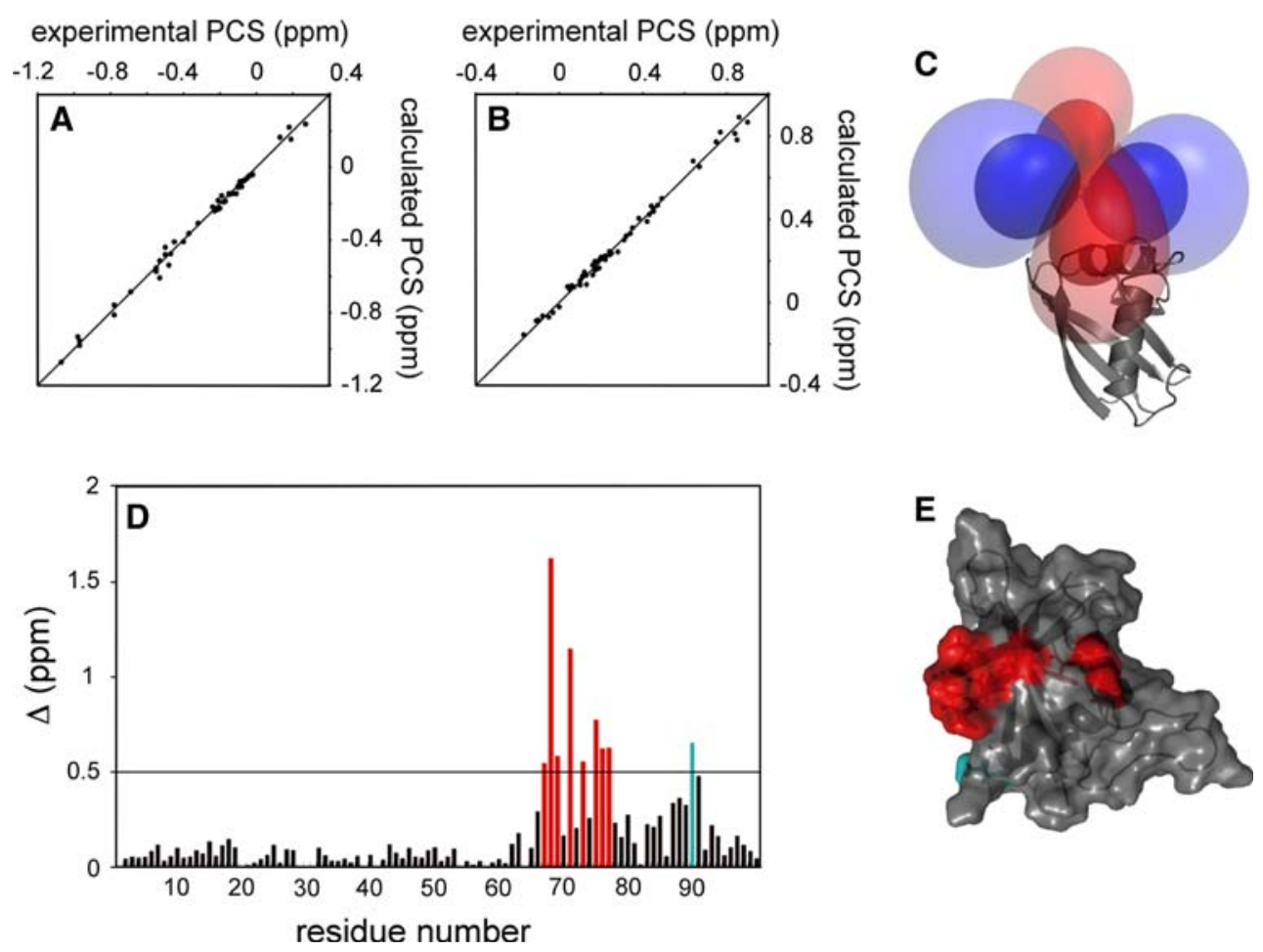

Fig. 3 Input parameter determination for the docking calculation. a and b Comparison of experimental and back-calculated PCSs of backbone amide protons observed for ${ }^{15} \mathrm{~N}$-labeled LBT-DR/unlabeled $\mathrm{KE}$ in complex with $\mathrm{Tb}^{3+}$ (a) and $\mathrm{Tm}^{3+}$ (b). The tensors were calculated using the monomer structure of DR. The ideal correlations are indicated. c View of the experimentally determined isosurfaces corresponding to a PCS of \pm 4.6 and $\pm 1.2 \mathrm{ppm}$, respectively. Positive and negative PCS values are indicated by blue and red, respectively. d Chemical shift perturbation of the backbone amide groups of $\mathrm{KE}$

Table $1 \Delta \chi$-tensor parameters for lanthanide ions in complex with LBT-DR/KE, determined on the basis of the monomer structure of DR and the PCS values obtained from LBT-DR signals

\begin{tabular}{lllll}
\hline & $\mathrm{Tb}^{3+}$ & $\mathrm{Tm}^{3+}$ & $\mathrm{Dy}^{3+}$ & $\mathrm{Er}^{3+}$ \\
\hline$\Delta \chi_{\mathrm{ax}}^{\mathrm{a}}$ & $40.8 \pm 1.1$ & $-27.2 \pm 1.3$ & $28.6 \pm 1.5$ & $-10.4 \pm 0.3$ \\
$\Delta \chi_{\mathrm{rh}}^{\mathrm{a}}$ & $20.7 \pm 0.9$ & $-18.9 \pm 1.0$ & $21.7 \pm 1.0$ & $-9.1 \pm 0.2$ \\
$\alpha^{\mathrm{b}}$ & 119 & 114 & 124 & 114 \\
$\beta^{\mathrm{b}}$ & 106 & 107 & 108 & 107 \\
$\gamma^{\mathrm{b}}$ & 15 & 2 & 37 & 14 \\
\hline
\end{tabular}

${ }^{\mathrm{a}} \Delta \chi_{\mathrm{ax}}$ and $\Delta \chi_{\mathrm{rh}}$ values are in $10^{-32}\left[\mathrm{~m}^{3}\right]$ and error estimates were obtained by Monte-Carlo protocol using the 100 partial PCS data sets in which $30 \%$ of the input data were randomly deleted. During the Monte-Carlo analysis, the metal position was allowed to vary within a range of $\pm 0.3 \AA$

${ }^{\mathrm{b}}$ Euler angle rotations in ZXZ convention (degrees)

perturbation information into the contact-surface restraints. It should be noted that the chemical shift perturbation mapping of LBT-DR, on binding with KE, indicated welldefined contact-surface area (Supporting information Figure S4). upon complex formation with LBT-DR at a ration of 1:1. $\Delta$ (ppm) was defined as $\left(\left(\Delta^{1} \mathrm{HN}\right)^{2}+\left(\Delta^{15} \mathrm{~N} / 5\right)^{2}\right)^{1 / 2}$ and plotted against the residue number of KE. The residues with $\Delta(\mathrm{ppm})>0.5$, except for Asp90, are shown in red. Asp90, which showed a chemical shift change larger than $0.5 \mathrm{ppm}$ but was not used as a contact surface restraint as it did not satisfy the inclusion criteria, is shown in cyan. $\mathbf{e}$ The mapping of the results with large chemical shift changes on the structure of p62 PB1. Residues are colored according to the color codes used in $(\mathbf{d})$

The contact-surface restraints were set up as distance restraints between the atoms of the selected residues of $\mathrm{KE}$ and all atoms of DR using the $r^{-6}$ averaging option (Ubbink et al. 1998; Clore and Schwieters 2003; DíazMoreno et al. 2005). For the $r^{-6}$ averaging option, the distance between selected sets of atoms is averaged according to the equation

$d=\left(\sum_{i j} r_{i j}^{-6}\right)^{-1 / 6}$,

where $r_{i j}$ represents the distance between the atom $i$ in the selected residue of $\mathrm{KE}$ and atom $j$ in all residues in DR. Averaging the minus 6 th power of the distance emphasizes the smaller distance values, thus a restraint is satisfied when at least one pair of the atoms locate close to each other.

Rigid-body docking

The docking calculation was carried out using the XplorNIH program (Schwieters et al. 2003, 2006) with a rigid body minimization protocol (Clore 2000; Tang and Clore 
2006). For the calculation, a total of 459 backbone ${ }^{1} \mathrm{H}$ and ${ }^{15} \mathrm{~N}$ PCS restraints derived from $\mathrm{Tb}^{3+}$ and $\mathrm{Tm}^{3+}$ as well as contact-surface restraints based on the chemical shift perturbation were used. The contact-surface restraints were added only to restrict the binding surface of KE. Details of the calculation are described in the "Method" section. A total of 100 structures were calculated, each of which started from the randomly arranged KE coordinates around DR. During the calculation, DR and the metal were held in a fixed position, while KE was freely rotated and translated as a rigid body. In the present calculation, we excluded Asp90 from the contact-surface restraints as it failed to satisfy all inclusion criteria (see above). However, test calculations showed that the inclusion of Asp90 in the contact-surface restraints had no effects on the results (data not shown). This may be due to the ambiguity of the distance restraints defined using the $r^{-6}$ averaging option.

The PCS-isosurface observed with a paramagnetic lanthanide ion is symmetric, thus a PCS data set derived from one lanthanide ion causes four degenerate solutions obtained by rotation around the $x, y$, and $z$ axes of the principal axis of the $\Delta \chi$-tensor. In principle, the degeneracy can be overcome by adding a second PCS data set from another lanthanide ion, as the direction of the principal axis of the $\Delta \chi$-tensor of a second lanthanide ion would be different from that of the first (Pintacuda et al. 2006). In our test calculations using only PCS restraints, the combined use of multiple PCS data sets couldn't overcome the degeneracy (data not shown), presumably due to the minute difference in the orientation of the principle axes of the $\Delta \chi$ tensors (Table 1). However, only one of the four degenerate solutions satisfied the contact surface restraints, thus a combination of the contact surface and PCS restraints allows the identification of a proper solution from among the four degenerate solutions.

An overlay of the 10 lowest energy structures and a ribbon model of the lowest energy structure of the DR/KE complex are shown in Fig. $4 \mathrm{a}$ and $\mathrm{b}$. These structures have an average backbone rmsd of $0.31 \AA$. The atomic coordinates and structural restraints for the DR/KE complex have been deposited in the Protein Data Bank, www.pdb.org (PDB code: 2KTR). The conserved acidic residues comprising the OPCA motif form two acidic patches, A1 (Asp67, Glu68, Asp69 and Asp71) and A2 (Glu80), and the basic residues around the conserved Lys motif similarly form B1 (Lys7, Arg22 and Arg94) and B2' (Arg21) patches (Fig. 4c). As shown in the open-book representation of the complex structure (Fig. 4c), A1 and A2 of KE interacts with $\mathrm{B} 1$ and $\mathrm{B} 2^{\prime}$ of DR, respectively. In addition to these electrostatic interactions, a hydrophobic interaction is also formed between Val73 of KE and Ile20 of DR, at the center of the interaction surface. This interaction mechanism of the p62 PB1-PB1 complex supports the previous notion
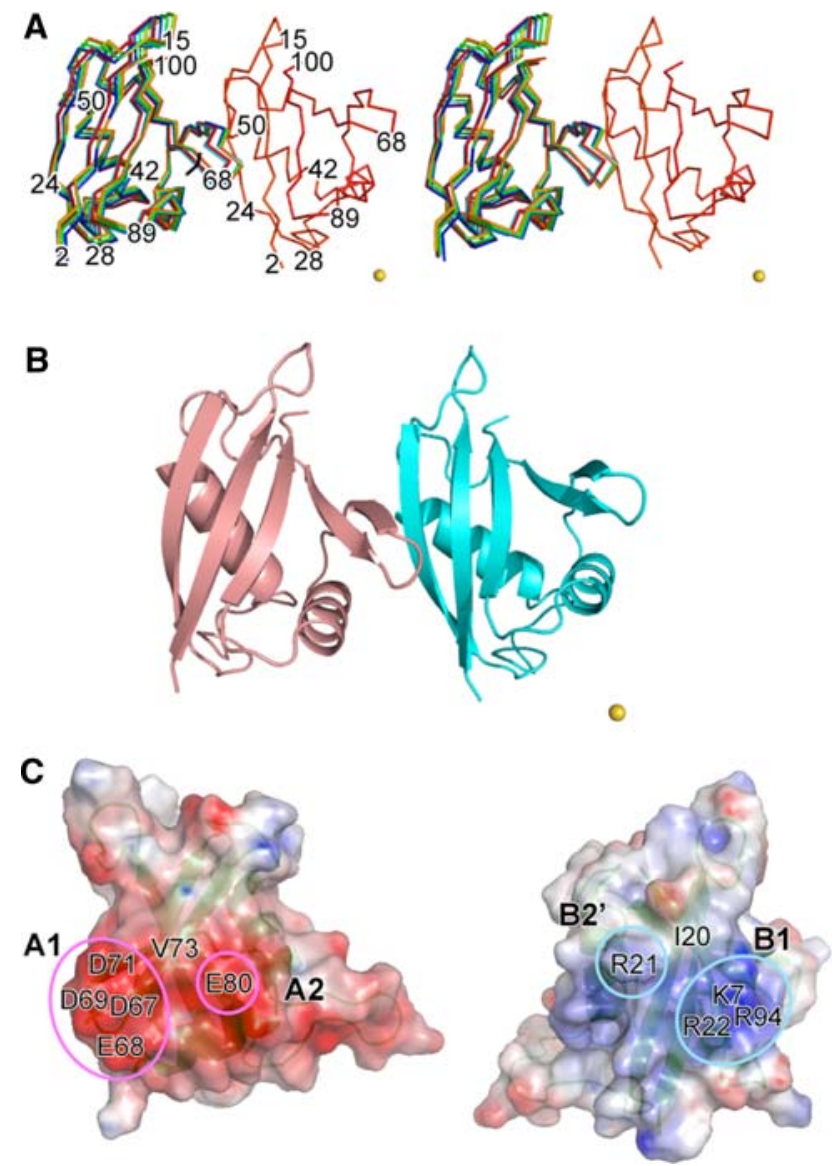

Fig. 4 The docking structure of the DR/KE complex. a Stereo view of the ensemble of the 10 lowest energy structures of the DR/KE complex. The structures of DR are superimposed. The metal position is represented as a yellow sphere. b Ribbon representation of the lowest energy structure. c Electrostatic surface potentials of the interaction surface of the DR/KE complex are shown in an open-book style. The conserved acidic and basic regions are circled in magenta and cyan, respectively. Positive and negative surface potentials are drawn in blue and red, respectively. The structures were drawn using the PyMOL program with APBS tools (http://www.pymol.org/)

that both electrostatic and hydrophobic interactions are involved in the PB1-PB1 complex formation (Ogura et al. 2009; Hirano et al. 2005; Wilson et al. 2003). It should be noted that the positions of $\mathrm{A} 1, \mathrm{~A} 2$, and Val73 of $\mathrm{KE}$ are complimentary to those of B1, B2', and Ile20 of DR, respectively.

Validation of the calculated structure

The docking structure of the DR/KE complex was independently validated using intermolecular NOEs observed in a sample prepared with ${ }^{15} \mathrm{~N} /{ }^{2} \mathrm{H}$-labeled LBT-DR and unlabeled KE. Representative examples of the intermolecular NOEs are listed in Table 2 and mapped on the PCSderived structure of DR/KE (Fig. 5a), and they can be seen to agree with the DR/KE structure described above. Several 
Table 2 Representative examples of intermolecular NOEs between LBT-DR and KE

\begin{tabular}{ll}
\hline Proton group in LBT-DR & Proton group on KE \\
\hline Ala8 $\mathrm{H}^{\mathrm{N}}$ & Val73 $\mathrm{C}^{\gamma 1} \mathrm{H}_{3}$ \\
Ala8 $\mathrm{H}^{\mathrm{N}}$ & Val73 $\mathrm{C}^{\gamma 2} \mathrm{H}_{3}$ \\
$\operatorname{Arg} 21 \mathrm{H}^{\mathrm{N}}$ & Val73 $\mathrm{C}^{\gamma 1} \mathrm{H}_{3}$ \\
$\operatorname{Arg} 21 \mathrm{H}^{\varepsilon}$ & Ala74 $\mathrm{C}^{\beta} \mathrm{H}_{2}$ \\
$\operatorname{Arg} 22 \mathrm{H}^{\mathrm{N}}$ & Val73 $\mathrm{C}^{\gamma 1} \mathrm{H}_{3}$ \\
$\operatorname{Arg} 22 \mathrm{H}^{\varepsilon}$ & Asp67 $\mathrm{C}^{\beta} \mathrm{H}_{2}$ \\
$\operatorname{Arg} 22 \mathrm{H}^{\varepsilon}$ & Glu68 $\mathrm{H}^{\mathrm{N}}$ \\
$\operatorname{Arg} 22 \mathrm{H}^{\varepsilon}$ & Met83 $\mathrm{C}^{\varepsilon} \mathrm{H}_{3}$ \\
Phe23 $\mathrm{H}^{\mathrm{N}}$ & Met83 $\mathrm{C}^{\varepsilon} \mathrm{H}_{3}$ \\
Ser24 $\mathrm{H}^{\mathrm{N}}$ & Met83 $\mathrm{C}^{\varepsilon} \mathrm{H}_{3}$ \\
\hline
\end{tabular}
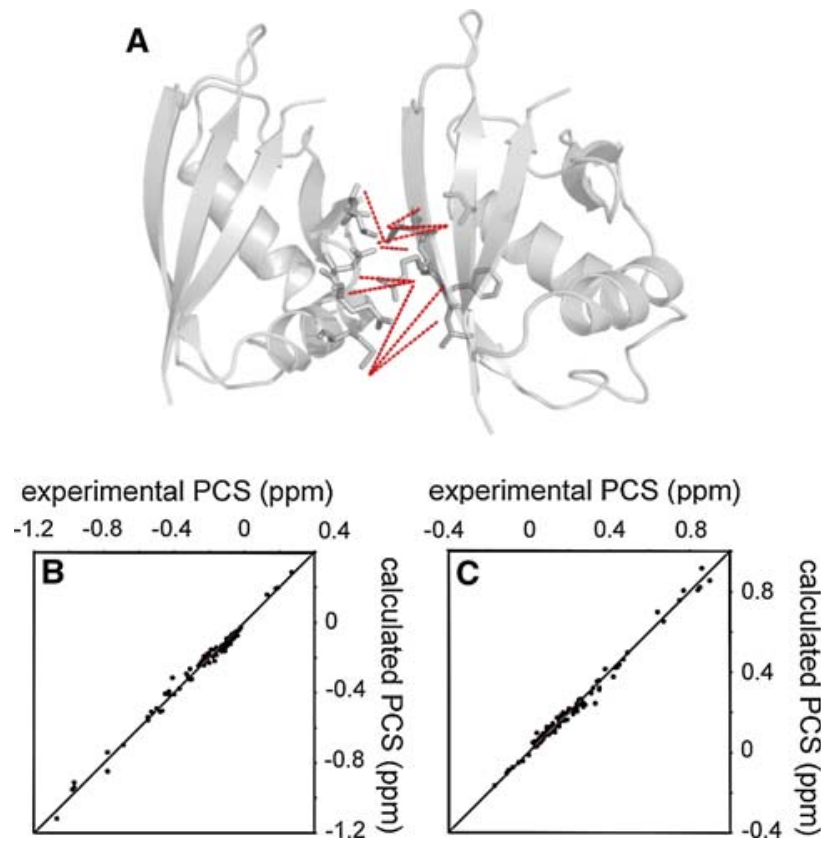

Fig. 5 Validation of the docking structure. a Intermolecular NOEs observed for ${ }^{15} \mathrm{~N} /{ }^{2} \mathrm{H}$-labeled LBT-DR complexed with unlabeled KE. The atoms between which NOEs were observed are displayed in a stick model and connected by dotted lines. The structure is shown in the direction rotated by $30^{\circ}$, along the longitudinal axis, from that in Fig. 4a and b. b and c Comparison of experimental and backcalculated PCSs of backbone amide protons observed for LBT-DR/ $\mathrm{KE}$ in complex with $\mathrm{Tb}^{3+}$ (b) and $\mathrm{Tm}^{3+}(\mathbf{c})$. The tensors were calculated using the whole lowest energy structure of the LBT-DR/ KE complex

NOEs were detected involving the Val73 of KE, which is located at the center of the interaction surface (Fig. 4c). Most of the intermolecular NOEs were observed between the atoms located at a distance of 4-6 $\AA$, whereas the distances between Arg22 $\mathrm{H}^{\varepsilon}$ and Met $83 \mathrm{C}^{\varepsilon} \mathrm{H}_{3}$, Phe $23 \mathrm{H}^{\mathrm{N}}$ and Met83 $\mathrm{C}^{\varepsilon} \mathrm{H}_{3}$, and Ser24 $\mathrm{H}^{\mathrm{N}}$ and Met83 $\mathrm{C}^{\varepsilon} \mathrm{H}_{3}$ were around $10 \AA$. This is explained by the side chain direction of the Met83. In conformer 1 of the family of NMR
Table $3 \Delta \chi$-tensor parameters for lanthanide ions in complex with LBT-DR/KE, determined on the basis of the docking structure of DR/ $\mathrm{KE}$ and the PCS values obtained both from LBT-DR and KE signals

\begin{tabular}{lllll}
\hline & $\mathrm{Tb}^{3+}$ & $\mathrm{Tm}^{3+}$ & $\mathrm{Dy}^{3+}$ & $\mathrm{Er}^{3+}$ \\
\hline$\Delta \chi_{\mathrm{ax}}^{\mathrm{a}}$ & $36.4 \pm 1.5$ & $-23.4 \pm 1.3$ & $21.9 \pm 0.8$ & $-10.0 \pm 0.4$ \\
$\Delta \chi_{\mathrm{rh}}^{\mathrm{a}}$ & $23.8 \pm 0.7$ & $-20.1 \pm 0.5$ & $20.4 \pm 0.7$ & $-9.9 \pm 0.3$ \\
$\alpha^{\mathrm{b}}$ & 117 & 108 & 120 & 119 \\
$\beta^{\mathrm{b}}$ & 103 & 105 & 104 & 103 \\
$\gamma^{\mathrm{b}}$ & 7 & 178 & 28 & 11 \\
\hline${ }^{\mathrm{a}} \Delta \chi_{\mathrm{ax}}$ and $\Delta \chi_{\mathrm{rh}}$ values are in $10^{-32}$ & {$\left[\mathrm{~m}^{3}\right]$ and error estimates were } \\
obtained by Monte-Carlo protocol using the 100 partial PCS data sets \\
in which 30\% of the input data were randomly deleted. During the \\
\multicolumn{4}{l}{ Monte-Carlo analysis, the metal position was allowed to vary within } \\
a range of $\pm 0.3 \AA$ \\
b Euler angle rotations in ZXZ convention (degrees)
\end{tabular}

structures of DR, used for the docking calculation, the methyl group of Met83 points in the opposite direction to the interaction surface, though it is closer to the interaction surface in another conformer.

We calculated the $\Delta \chi$-tensor parameters based on the docking structure and the PCS values obtained both from LBT-DR and KE (Table 3 and supporting information Figure S5). The $\Delta \chi$-tensor parameters were well defined and, furthermore, comparable to those determined for LBT-DR (Table 1 and supporting information Figure S2). It should be noted that correlations between the experimental and back-calculated PCS values were good, which also supports the compatibility of the docking structure (Fig. 5b, c, and supporting information Figure S6). The magnitudes of $\Delta \chi$-tensors calculated for the KE part alone were similar to those for LBT-DR and the LBT-DR/KE complex, which suggests stable complex formation between LBT-DR and KE (Supporting information Table S2). We also observed RDCs for LBT-DR/KE containing $\mathrm{Tm}^{3+}$. The magnitudes of the RDC values observed for KE were up to $14 \mathrm{~Hz}$ using $800 \mathrm{MHz} \mathrm{NMR}$ at $25^{\circ} \mathrm{C}$, which were nearly identical to those observed for LBT-DR. This also supports the stability of the LBT-DR/KE complex.

\section{Conclusion}

A paramagnetic lanthanide ion provides valuable information for NMR protein structural analysis as PCS contains both long-range distance and angular information, which cannot be replaced by other probes, such as spin labels, NOEs, or paramagnetic metal ions (e.g., $\mathrm{Cu}^{2+}$, $\mathrm{Mn}^{2+}$, or $\mathrm{Gd}^{3+}$ ), that only yield distance dependent information. Bertini et al. (2009) demonstrated accurate solution structure determinations of multi-domain metalloproteins utilizing paramagnetic lanthanide probes. Despite the advantages associated with the use of lanthanide probes, 
the application of this approach has been limited to certain metal-binding proteins. To apply this method to non metalbinding proteins, a wide variety of lanthanide ion anchoring tags have been developed, including lanthanide binding peptide tags and synthetic chelating reagents ( $\mathrm{Su}$ and Otting 2009). However, lanthanide tagging has not yet been applied to protein structural analysis apart from a limited number of studies (Gaponenko et al. 2002, 2004; Zhuang et al. 2008; Xu et al. 2009). Recently, Feng et al. (2007) reported the structural analysis on the homo-oligomeric domain, Par-3 NTD, and utilized the lanthanide tagging method to obtain structural information for the complex. However, the high mobility of the lanthanide ion prevented the quantitative analysis of PCS. In general, the flexibility of the lanthanide binding tag prevents the wider application of lanthanide probes.

Recently, we reported a two-point anchoring method for a lanthanide binding peptide tag that fulfills both the need for ready availability for protein NMR researchers and higher rigidity (Saio et al. 2009a). Using this lanthanide tagging method, we here determined the protein-protein complex structure of the p62 PB1 homo-dimer, based on the distance and angular restraints from backbone ${ }^{1} \mathrm{H} /{ }^{15} \mathrm{~N}$ PCSs and the contact-surface restraints derived from backbone chemical shift perturbations. These two kinds of restraints can easily be obtained by the measurement of ${ }^{1} \mathrm{H}^{-15} \mathrm{~N}$ HSQC spectra, as long as the backbone assignment of the target is available. On the other hand, NOE-based methods require experiments, far less sensitive than ${ }^{1} \mathrm{H}-{ }^{15} \mathrm{~N}$ HSQC spectra, for side chain assignment and NOE collection, which is time-consuming and often difficult, especially for larger molecular weight targets. Using the two-point anchored peptide tag, which is readily available and holds a lanthanide ion in a fixed position, the application of the paramagnetic lanthanide probe will become more widely used.

Open Access This article is distributed under the terms of the Creative Commons Attribution Noncommercial License which permits any noncommercial use, distribution, and reproduction in any medium, provided the original author(s) and source are credited.

\section{References}

Allegrozzi M, Bertini I, Janik MBL, Lee YM, Liu G, Luchinat C (2000) Lanthanide-induced pseudocontact shifts for solution structure refinements of macromolecules in shells up to $40 \AA$ from the metal ion. J Am Chem Soc 122:4154-4161

Banci L, Bertini I, Cavallaro G, Giachetti A, Luchinat C, Parigi G (2004) Paramagnetism-based restraints for Xplor-NIH. J Biomol NMR 28:249-261

Barbieri R, Bertini I, Cavallaro G, Lee YM, Luchinat C, Rosato A (2002) Paramagnetically induced residual dipolar couplings for solution structure determination of lanthanide binding proteins. J Am Chem Soc 124:5581-5587

Bertini I, Janik MB, Liu G, Luchinat C, Rosato A (2001) Solution structure calculations through self-orientation in a magnetic field of a cerium(III) substituted calcium-binding protein. J Magn Reson 148:23-30

Bertini I, Del Bianco C, Gelis I, Katsaros N, Luchinat C, Parigi G, Peana M, Provenzani A, Zoroddu MA (2004) Experimentally exploring the conformational space sampled by domain reorientation in calmodulin. Proc Natl Acad Sci USA 101:6841-6846

Bertini I, Gupta YK, Luchinat C, Parigi G, Peana M, Sgheri L, Yuan J (2007) Paramagnetism-based NMR restraints provide maximum allowed probabilities for the different conformations of partially independent protein domains. J Am Chem Soc 129:12786-12794

Bertini I, Kursula P, Luchinat C, Parigi G, Vahokoski J, Wilmanns M, Yuan J (2009) Accurate solution structures of proteins from Xray data and a minimal set of NMR data: calmodulin-peptide complexes as examples. J Am Chem Soc 131:5134-5144

Bjørkøy G, Lamark T, Brech A, Outzen H, Perander M, Overvatn A, Stenmark H, Johansen T (2005) p62/SQSTM1 forms protein aggregates degraded by autophagy and has a protective effect on huntingtin-induced cell death. J Cell Biol 171:603-614

Clore GM (2000) Accurate and rapid docking of protein-protein complexes on the basis of intermolecular nuclear overhauser enhancement data and dipolar couplings by rigid body minimization. Proc Natl Acad Sci USA 97:9021-9025

Clore GM, Schwieters CD (2003) Docking of protein-protein complexes on the basis of highly ambiguous intermolecular distance restraints derived from $1 \mathrm{H} / 15 \mathrm{~N}$ chemical shift mapping and backbone $15 \mathrm{~N}-1 \mathrm{H}$ residual dipolar couplings using conjoined rigid body/torsion angle dynamics. J Am Chem Soc 125:2902-2912

Delaglio F, Grzesiek S, Vuister G, Zhu W, Pfeifer J, Bax A (1995) NMRPipe: a multidimensional spectral processing system based on UNIX pipes. J Biomol NMR 6:277-293

Díaz-Moreno I, Díaz-Quintana A, De la Rosa MA, Ubbink M (2005) Structure of the complex between plastocyanin and cytochrome $f$ from the cyanobacterium Nostoc sp. PCC 7119 as determined by paramagnetic NMR. The balance between electrostatic and hydrophobic interactions within the transient complex determines the relative orientation of the two proteins. J Biol Chem 280:18908-18915

Dvoretsky A, Gaponenko V, Rosevear PR (2002) Derivation of structural restraints using a thiol-reactive chelator. FEBS Lett 528:189-192

Feng W, Wu H, Chan LN, Zhang M (2007) The Par-3 NTD adopts a PB1-like structure required for Par-3 oligomerization and membrane localization. EMBO J 26:2786-2796

Gaponenko V, Dvoretsky A, Walsby C, Hoffman BM, Rosevear PR (2000) Calculation of $z$-coordinates and orientational restraints using a metal binding tag. Biochemistry 39:15217-15224

Gaponenko V, Altieri AS, Li J, Byrd RA (2002) Breaking symmetry in the structure determination of (large) symmetric protein dimers. J Biomol NMR 24:143-148

Gaponenko V, Sarma SP, Altieri AS, Horita DA, Li J, Byrd RA (2004) Improving the accuracy of NMR structures of large proteins using pseudocontact shifts as long-range restraints. J Biomol NMR 28:205-212

Haberz P, Rodriguez-Castañeda F, Junker J, Becker S, Leonov A, Griesinger C (2006) Two new chiral EDTA-based metal chelates for weak alignment of proteins in solution. Org Lett 8:12751278

Hirano Y, Yoshinaga S, Takeya R, Suzuki NN, Horiuchi M, Kohjima M, Sumimoto H, Inagaki F (2005) Structure of a cell polarity regulator, a complex between atypical PKC and Par6 PB1 domains. J Biol Chem 280:9653-9661 
Ikegami T, Verdier L, Sakhaii P, Grimme S, Pescatore B, Saxena K, Fiebig KM, Griesinger C (2004) Novel techniques for weak alignment of proteins in solution using chemical tags coordinating lanthanide ions. J Biomol NMR 29:339-349

Keizers PH, Desreux JF, Overhand M, Ubbink M (2007) Increased paramagnetic effect of a lanthanide protein probe by two-point attachment. J Am Chem Soc 129:9292-9293

Keizers PH, Saragliadis A, Hiruma Y, Overhand M, Ubbink M (2008) Design, synthesis, and evaluation of a lanthanide chelating protein probe: CLaNP-5 yields predictable paramagnetic effects independent of environment. J Am Chem Soc 130:14802-14812

Leonov A, Voigt B, Rodriguez-Castañeda F, Sakhaii P, Griesinger C (2005) Convenient synthesis of multifunctional EDTA-based chiral metal chelates substituted with an S-mesylcysteine. Chem Eur J 11:3342-3348

Ma C, Opella SJ (2000) Lanthanide ions bind specifically to an added "EF-hand" and orient a membrane protein in micelles for solution NMR spectroscopy. J Magn Reson 146:381-384

Martin LJ, Hähnke MJ, Nitz M, Wöhnert J, Silvaggi NR, Allen KN, Schwalbe H, Imperiali B (2007) Double-lanthanide-binding tags: design, photophysical properties, and NMR applications. J Am Chem Soc 129:7106-7113

Nitz M, Franz KJ, Maglathlin RL, Imperiali B (2003) A powerful combinatorial screen to identify high-affinity terbium(III)-binding peptides. Chembiochem 4:272-276

Nitz M, Sherawat M, Franz KJ, Peisach E, Allen KN, Imperiali B (2004) Structural origin of the high affinity of a chemically evolved lanthanide-binding peptide. Angew Chem Int Ed Engl 12:3682-3685

Noda NN, Kumeta H, Nakatogawa H, Satoo K, Adachi W, Ishii J, Fujioka Y, Ohsumi Y, Inagaki F (2008) Structural basis of target recognition by Atg8/LC3 during selective autophagy. Genes Cells 13:1211-1218

Ogura K, Tandai T, Yoshinaga S, Kobashigawa Y, Kumeta H, Ito T, Sumimoto H, Inagaki F (2009) NMR structure of the heterodimer of Bem1 and Cdc24 PB1 domains from Saccharomyces cerevisiae. J Biochem 146:317-325

Pintacuda G, Moshref A, Leonchiks A, Sharipo A, Otting G (2004) Site-specific labelling with a metal chelator for protein-structure refinement. J Biomol NMR 29:351-361

Pintacuda G, Park AY, Keniry MA, Dixon NE, Otting G (2006) Lanthanide labeling offers fast NMR approach to 3D structure determinations of protein-protein complexes. J Am Chem Soc 128:3696-3702

Pintacuda G, John M, Su XC, Otting G (2007) NMR structure of protein-ligand complexes by lanthanide labeling. Acc Chem Res 40:206-212

Prudêncio M, Rohovec J, Peters JA, Tocheva E, Boulanger MJ, Murphy ME, Hupkes HJ, Kosters W, Impagliazzo A, Ubbink M (2004) A caged lanthanide complex as a paramagnetic shift agent for protein NMR. Chemistry 10:3252-3260

Qoronfleh MW, Hesterberg LK, Seefeldt MB (2007) Confronting high-throughput protein refolding using high pressure and solution screens. Protein Expr Purif 55:209-224

Saio T, Ogura K, Yokochi M, Kobashigawa Y, Inagaki F (2009a) Two-point anchoring of a lanthanide-binding peptide to a target protein enhances the paramagnetic anisotropic effect. J Biomol NMR 44:157-166

Saio T, Yokochi M, Inagaki F (2009b) The NMR structure of the p62 $\mathrm{PB} 1$ domain, a key protein in autophagy and NF- $\kappa \mathrm{B}$ signaling pathway. J Biomol NMR (in press)

Schmitz C, Stanton-Cook MJ, Su XC, Otting G, Huber T (2008) Numbat: an interactive software tool for fitting Deltachi-tensors to molecular coordinates using pseudocontact shifts. J Biomol NMR 41:179-189

Schoner BE, Bramlett KS, Guo H, Burris TP (2005) Reconstitution of functional nuclear receptor proteins using high pressure refolding. Mol Genet Metab 85:318-322

Schwieters CD, Kuszewski JJ, Tjandra N, Clore GM (2003) The Xplor-NIH NMR molecular structure determination package. J Magn Reson 160:65-73

Schwieters CD, Kuszewski JJ, Clore GM (2006) Using Xplor-NIH for NMR molecular structure determination. Progr Nucl Magn Resn Spectr 48:47-62

Su XC, Otting G (2009) Paramagnetic labelling of proteins and oligonucleotides for NMR. J Biomol NMR. (ahead of print)

Su XC, Huber T, Dixon NE, Otting G (2006) Site-specific labelling of proteins with a rigid lanthanide-binding tag. ChemBioChem 7:1599-1604

Su XC, McAndrew K, Huber T, Otting G (2008a) Lanthanide-binding peptides for NMR measurements of residual dipolar couplings and paramagnetic effects from multiple angles. J Am Chem Soc 130:1681-1687

Su XC, Man B, Beeren S, Liang H, Simonsen S, Schmitz C, Huber T, Messerle BA, Otting G (2008b) A dipicolinic acid tag for rigid lanthanide tagging of proteins and paramagnetic NMR spectroscopy. J Am Chem Soc 130:10486-10487

Tang C, Clore GM (2006) A simple and reliable approach to docking protein-protein complexes from very sparse NOE-derived intermolecular distance restraints. J Biomol NMR 36:37-44

Ubbink M, Ejdebäck M, Karlsson BG, Bendall DS (1998) The structure of the complex of plastocyanin and cytochrome $f$, determined by paramagnetic NMR and restrained rigid-body molecular dynamics. Structure 6:323-335

Vlasie MD, Comuzzi C, van den Nieuwendijk AM, Prudêncio M, Overhand M, Ubbink M (2007) Long-range-distance NMR effects in a protein labeled with a lanthanide-DOTA chelate. Chem Eur J 13:1715-1723

Wilson MI, Gill DJ, Perisic O, Quinn MT, Williams RL (2003) PB1 domain-mediated heterodimerization in NADPH oxidase and signaling complexes of atypical protein kinase $\mathrm{C}$ with Par6 and p62. Mol Cell 12:39-50

Wöhnert J, Franz KJ, Nitz M, Imperiali B, Schwalbe H (2003) Protein alignment by a coexpressed lanthanide-binding tag for the measurement of residual dipolar couplings. J Am Chem Soc 125:13338-13339

Xu X, Keizers PH, Reinle W, Hannemann F, Bernhardt R, Ubbink M (2009) Intermolecular dynamics studied by paramagnetic tagging. J Biomol NMR 43:247-254

Zhuang T, Lee HS, Imperiali B, Prestegard JH (2008) Structure determination of a galectin-3-carbohydrate complex using paramagnetism-based NMR constraints. Protein Sci 17:1220-1231 